\title{
ORIGINAL
}

\section{EVOLUCIÓN DE LA MORTALIDAD ATRIBUIBLE AL TABACO EN LAS ISLAS CANARIAS (1975-1994)}

Luis M Bello Luján (1), Pedro Lorenzo Ruano (1), Mercedes Gil Muñoz (1), Pedro Saavedra Santana (2) y Lluis Serra Majem (3)

(1) Dirección General de Salud Pública del Gobierno de Canarias.

(2) Departamento de Matemáticas. Universidad de las Palmas de Gran Canaria.

(3) Cátedra de Medicina Preventiva. Universidad de Las Palmas de Gran Canaria.

\section{RESUMEN}

Fundamento: El objetivo de este trabajo es describir la mortalidad atribuible al consumo de tabaco en las Islas Canarias durante el período 1975-1994.

Método: Las defunciones por edad, sexo y causa desde 1975 a 1994 se obtuvieron del Instituto Nacional de Estadística (Movimiento Natural Población). A partir de las Encuestas de Salud de España y Canarias se tomaron los porcentajes de nunca fumadores, fumadores y ex-fumadores de la población canaria por edad y sexo. Los riesgos relativos de muerte se obtuvieron del Cancer Prevention Study II, llevado a cabo en Estados Unidos de América. Se calculó la proporción de muertes atribuibles al tabaco para cada año, sexo y grupo de edad a partir de la fracción atribuible poblacional. Así mismo, se calculó la tendencia de la mortalidad atribuible para dicho período expresada como el cambio porcentual medio anual de las tasas de la mortalidad ajustadas por edad, mediante un modelo log-lineal.

Resultados: Durante el período 1975-1994, el número de fallecimientos atribuidos al tabaco aumentó un $64 \%$. Por grandes causas, se observó en el período de estudio, un aumento de las neoplasias del 108\%, una disminución de las enfermedades cardiovasculares del $32 \%$ y un incremento de las enfermedades respiratorias del $15,5 \%$. Se observó también que el número de fallecidos aumenta con la edad, siendo el grupo de edad de 65 años y más en el que se presentan más muertes por el tabaco.

Conclusiones: En las Islas Canarias, más del 20\% de todas las muertes en 1994 se pueden atribuir al tabaco. Esto sugiere que las medidas introducidas para controlar el tabaquismo son insuficientes.

Palabras claves: Tabaco. Mortalidad atribuible. Islas Canarias.

Correspondencia:

Luis M Bello Luján

C/ Pedro Cerón 13-1. ${ }^{\circ}$

35001 Las Palmas de Gran Canaria

Correo electrónico: lbello@dgsp.scstf.rcanaria.es
ABSTRACT

\section{Trend in the Mortality Attributable to Tobacco on the Canary Islands (1975-1994)}

Background: The objective of this study is that of describing the death rate attributable to tobacco on the Canary Islands throughout the 1975-1994 period.

Method: Deaths by age, sex and cause from 1975 to 1994 were obtained from the Spanish National Institute of Statistics (Natural Movement of Population). Based on the Spanish and Canary Island Health Surveys, the percentages of those who had never smoked, smokers and ex-smokers for the Canary Island population were taken by age and sex. The relative risks of death were taken from the Cancer Prevention Study II carried out in the United States. The percentages of deaths attributable to smoking were calculated for each year, sex and age group based on the attributable fraction of the population. Likewise, the trend in the death rate attributable for the time period in question was calculated and given in the form of the annual mean percentage change in the age-adjusted death rates by way of a log-linear model

Results: During the 1975-1994 period, the number of deaths attributed to smoking rose by $64 \%$. For major causes, a $108 \%$ increase in neoplasias, a $32 \%$ drop in cardiovascular diseases and a $15.5 \%$ increase in respiratory diseases were found for the period under study. The number of deaths was also found to increase with age, the 65 and over age group having been found to be that in which the most deaths caused by smoking occurred.

Conclusions: On the Canary Islands, over $20 \%$ of all deaths in 1994 can be attributed to smoking. This suggests that the measures implemented to control the smoking habit are insufficient.

Keywords: Tobacco. Attributable mortality. Canary Islands. 


\section{INTRODUCCIÓN}

El consumo de tabaco es la principal causa de morbimortalidad evitable y prematura tanto en los Estados Unidos como en Europa. Solo en Estados Unidos, más de 400.000 personas fallecen cada año como consecuencia del tabaco ${ }^{1}$. En España (1992) se llegó a una cifra de 46.226 fallecidos atribuibles al tabaco ${ }^{2}$. En Madrid (1995) a 34.252 fallecidos $^{3}$ y en las Islas Canarias (1995) a 1500 fallecidos atribuibles al tabaco en un solo año ${ }^{4}$.

Diversas causas de defunción se han asociado con el consumo de tabaco, entre las que se encuentran diferentes tipos de cáncer, enfermedades cardiovasculares, respiratorias y enfermedades en los recién nacidos (bajo peso al nacer, síndrome de distréss respiratorio, y síndrome de la muerte súbita) $)^{5-7}$.

Según la Encuesta Nacional de Salud (1993) se obtuvo una prevalencia del consumo de tabaco del $36 \%$ para Canarias $(47,5 \%$ en varones y $25,6 \%$ mujeres), cifra similar a la de España ${ }^{8}$, siendo ligeramente inferior a la prevalencia del año 1987 , que fue de $37,2 \%(50,8 \%$ en varones y $23,6 \%$ en mujeres) obtenida también de la Encuesta Nacional de Salud ${ }^{9}$. Según la Encuesta de Salud de Canarias (1990) ${ }^{10}$ la prevalencia del consumo de tabaco en la población canaria estuvo en torno al 30,3\% en el año 1990; en cambio, en la del año $1997^{11}$ nos reflejó un ligero incremento de misma (33\%) como consecuencia de la incorporación de la mujer, que ha pasado de un 19,4\% en 1990 a un 24,6\% en 1997.

Si bien se ha podido disponer en las Islas Canarias de datos sobre el consumo de tabaco a partir de las diferentes encuestas de salud que se han llevado a cabo, no se disponen de estudios epidemiológicos sobre una serie tan larga para conocer cuál ha sido el impacto que dicho consumo ha tenido en la mortalidad de la población en las islas. Por tanto, en este estudio se pretende conocer cuál es el número de muertes atribuidas al consumo de tabaco para el período 1975-1994, así como el cambio que se ha producido en las tasas de mortalidad para cada causa y en ese período en las Islas Canarias.

\section{MATERIAL Y MÉTODOS}

Defunciones. El número de defunciones por edad (35 y más años), sexo y causa de muerte correspondientes a los códigos de la 8. ${ }^{a}$ y 9. ${ }^{a}$ revisión de la Clasificación Internacional de Enfermedades (CIE) se obtuvieron de los ficheros individualizados de defunciones por causas de muerte del Instituto Nacional de Estadística (INE) correspondientes a las Islas Canarias de los años 1975 a 1994. Las causas de muerte que se han incluido han sido aquellas para las que existe una evidencia consistente de asociación con el consumo de tabaco.

Poblaciones. Las poblaciones utilizadas para el cálculo de las tasas de mortalidad son las que corresponden entre 1975 y 1994 estimadas por el Instituto Canario de Estadística (INSTAC) para las Islas Canarias.

Magnitud del efecto. Se utilizaron los riesgos relativos en el estudio de cohortes prospectivo Cancer Prevention Study II (tabla 1). En este estudio se incluyó a los adultos mayores de 30 años, de raza blanca y de clase media ${ }^{12}$.

Prevalencia de consumo de tabaco. Se consideraron las prevalencias de fumadores, ex-fumadores y nunca fumadores por sexo y edad (35-44, 45-64 y 65 y más años) obtenidas de la fracción para las Islas Canarias de las Encuesta Nacional Salud de España/19879, la del año $1993^{8}$ y de las Encuesta Salud de Canarias $/ 1990^{10}$ y la del año $1997^{11}$. Para la década 1970-79 se ha asumido que la proporción de varones fumadores a lo largo de la década fue del $60 \%$ y de mujeres el $15 \%{ }^{13}$. Para los años en los cuales no se dispuso de datos reales de prevalencias para Canarias se realizaron estimaciones de 
Tabla 1

Riesgos relativos de muerte debidos a enfermedades relacionadas con el con el tabaquismo en los fumadores y ex-fumadores según sexo

\begin{tabular}{|c|c|c|c|c|c|}
\hline \multirow{2}{*}{$\begin{array}{l}\text { CAUSAS DE MUERTE } \\
\quad \text { (adultos }>35 \text { años) }\end{array}$} & \multirow{2}{*}{$\begin{array}{l}C O D I G O S \\
C I E / 8 .^{a}-9{ }^{a}\end{array}$} & \multicolumn{2}{|c|}{$\begin{array}{l}R R(*) \\
H O M B R E S\end{array}$} & \multicolumn{2}{|c|}{$\begin{array}{c}R R(*) \\
\text { MUJERES }\end{array}$} \\
\hline & & Fumadores & Ex-Fumadores & Fumadores & Ex-Fumadores \\
\hline \multicolumn{6}{|l|}{ NEOPLASIAS } \\
\hline Labio, boca, faringe & $140-149$ & 27,5 & 8,8 & 5,6 & 2,9 \\
\hline Esófago & 150 & 7,6 & 5,8 & 10,3 & 3,2 \\
\hline Páncreas & 157 & 2,1 & 1,1 & 2,3 & 1,8 \\
\hline Laringe & 161 & 10,5 & 5,2 & 17,8 & 11,9 \\
\hline Tráquea, bronquios, pulmón & 162 & 22,4 & 9,4 & 11,9 & 4,7 \\
\hline Cuello de útero & 180 & n.a. & n.a. & 2,1 & 1,9 \\
\hline Vejiga & 188 & 2,9 & 1,9 & 2,6 & 1,9 \\
\hline Riñón & 189 & 3 & 2 & 1,4 & 1,2 \\
\hline \multicolumn{6}{|l|}{ ENF. CARDIOVASCULARES } \\
\hline Enfermedad hipertensiva & $400-404,401-405$ & 1,9 & 1,3 & 1,7 & 1,2 \\
\hline Cardiopatía isquémica & $410-414$ & & & & \\
\hline 35-64 años & & 2,8 & 1,8 & 3 & 1,4 \\
\hline$>=65$ años & & 1,6 & 1,3 & 1,6 & 1,3 \\
\hline \multirow{2}{*}{ Otras Enfermedades del aparato circulatorio } & $393-398,415-417$ & 1,9 & 1,3 & 1,7 & 1,2 \\
\hline & $420-429$ & & & & \\
\hline Enfermedad cerebro-vascular & $430-438$ & & & & \\
\hline 35-64 años & & 3,7 & 1,4 & 4,8 & 1,4 \\
\hline$>=65$ años & & 1,9 & 1,3 & 1,5 & 1 \\
\hline Aterosclerosis & 440 & 4,1 & 2,3 & 3 & 1,3 \\
\hline Aneurisma aórtico & 441 & 4,1 & 2,3 & 3 & 1,3 \\
\hline $\begin{array}{l}\text { Otras Enfermedades de arterias, arteriolas y } \\
\text { capilares }\end{array}$ & $442-448$ & 4,1 & 2,3 & 3 & 1,3 \\
\hline \multicolumn{6}{|l|}{ ENF. RESPIRATORIAS } \\
\hline Neumonía e Influenza & $480-487$ & 2 & 1,6 & 2,2 & 1,4 \\
\hline Bronquitis crónica y Enfisema & $491-492$ & 9,7 & 8,8 & 10,5 & 7 \\
\hline Obstrucción crónica de vías respiratorias & $496-493$ & 9,7 & 8,8 & 10,5 & 7 \\
\hline Otras Enfermedades Respiratorias & $010-012,493$ & 2 & 1,6 & 2,2 & 1,4 \\
\hline
\end{tabular}

$(*)$ : Riesgo relativo

las mismas asumiendo incrementos o decrementos anuales constantes durante los períodos intermedios entre los años correspondientes a las encuestas citadas ${ }^{2}$.

Cálculo de muertes atribuibles. Se obtuvo la proporción de muertes atribuibles al consumo de tabaco en la población para cada año, causa de muerte, sexo y grupo de edad, teniendo en cuenta la formula siguiente:

$$
\begin{gathered}
\mathrm{FAP}=\left[\mathrm{p}_{0}+\mathrm{p}_{1}\left(\mathrm{RR}_{1}\right)+\mathrm{p}_{2}\left(\mathrm{RR}_{2}\right)\right]-1 /\left[\mathrm{p}_{0}+\right. \\
+\mathrm{p}_{1}\left(\mathrm{RR}_{1}\right)+\mathrm{p}_{2}\left(\mathrm{RR}_{2}\right)
\end{gathered}
$$

FAP: Fracción atribuible poblacional; $\mathrm{P}_{0}$ : Proporción de población nunca fumadora; $\mathrm{P}_{1}$ : Proporción de población fumadora; $\mathrm{P}_{2}$ : Proporción de población ex-fumadora; $\mathrm{RR}_{1}$ : Riesgo relativo en población fumadora; $\mathrm{RR}_{2}$ : Riesgo relativo en población ex-fumadora.

Las muertes atribuibles al consumo de tabaco se han obtenido al aplicar esta proporción a las muertes ocurridas para cada año y causa. Además se ha calculado la mortalidad ajustada por edad (método directo) para 
cada causa y año. Para el ajuste de las tasas se utilizó la población europea como estándar.

Mediante el cambio medio anual de las tasas ajustadas de mortalidad atribuible al consumo de tabaco se calculó la tendencia de la mortalidad atribuible al tabaco según un modelo log-lineal.

$\log ={ }_{0}+\operatorname{gedad}+{ }_{\mathrm{s}}$ sexo $+{ }_{\mathrm{p}}$ período

donde representa el valor esperado de la variable $F A P$, la cual supone que sigue una distribución probabilística de Poisson. La variable ordinal gedad corresponde a los diferentes grupos de edad, sexo es una variable binaria con el valor uno o cero según el grupo sea de hombres o mujeres y periodo es una variable numérica cuyos valores corresponden a los años considerados. A partir del modelo log-lineal (1), la proporción anual de aumento de mortalidad se calcula por $p^{-1}$. Este modelo (1) se ha estimado utilizando el paquete estadístico $\mathrm{SAS}^{14}$.

\section{RESULTADOS}

En 1975 se produjo un total de 1.551 fallecidos atribuidos al consumo de tabaco, lo que representó casi el 20\% de todos los fallecidos para ese año. En cambio, en 1994 el total fue de 2.262 lo que representó el 24\% de todos los fallecidos de las Islas Canarias. Durante el período 1975-94 se produjo un incremento del $64 \%$ en el número de falleci- dos atribuibles al tabaco. En 1975, el 73,7\% de los fallecidos correspondieron a varones y el $26,2 \%$ a mujeres y en 1994 , el $75,5 \%$ y el $24,4 \%$, respectivamente.

Por grandes causas, en 1975 se observa que el 19,5\% de los fallecidos atribuidos al tabaco se debió a las neoplasias, el 70,2\% a las enfermedades cardiovasculares y el $10,2 \%$ a las enfermedades respiratorias; en 1994 , los porcentajes fueron $32,0 \%, 52,7 \%$, $14,2 \%$ respectivamente (tabla 2 , figura 1 ).

Por causas específicas, en 1994 se observa que el $20.8 \%$ de las muertes atribuidas al tabaco se deben a la cardiopatía isquémica (472), el $18,6 \%$ a la neoplasia de tráquea, bronquios y pulmón (413), el 12,8 \% a la enfermedad cerebro-vascular (291) y el 8,7\% a la obstrucción de vías aéreas (198). Por sexo, en hombres el $22,0 \%$ de los fallecimientos atribuidos al tabaco se deben a la neoplasia de tráquea, bronquios y pulmón (376), el 20,2\% a la cardiopatía isquémica (347), el 10,7 a la enfermedad cerebro-vascular (185) y el $9,3 \%$ a la obstrucción de vías respiratorias (160); en mujeres, el 22,4\% de los fallecidos atribuidos al tabaco se deben a la cardiopatía isquémica (125), el 19\% a la enfermedad cerebro-vascular (106), el 6,8\% a la obstrucción de vías aéreas (38) y el 6,6\% a la neoplasia de tráquea, bronquios y pulmón (37). Solo estas cuatro causas específicas suman el 60,9\% de los fallecidos atribuidos al tabaco; en hombres, el $62,2 \%$ y en las mujeres el 54,8\% (tabla 3 , figura 2).

Tabla 2

Porcentaje de fallecidos atribuibles al Tabaco en personas de 35 y más años

\begin{tabular}{|lllllrr|}
\hline & \multicolumn{2}{c}{ Totales } & \multicolumn{3}{c}{ Hombres } & \multicolumn{2}{c|}{ Mujeres } \\
\hline & 1975 & 1994 & 1975 & 1994 & 1975 & 1994 \\
\hline Neoplasias (140-189) & 19,5 & 33 & 23,5 & 38,9 & 8,3 & 14,6 \\
Enfermedades cardiovasculares (401-448) & 70,2 & 52,7 & 67 & 46,9 & 79,1 & 70,7 \\
Enfermedades Respiratorias (480-493) & 10,2 & 14,2 & 9,4 & 14,1 & 12,5 & 14,6 \\
\hline
\end{tabular}

Fuente: Instituto Nacional Estadística (Movimiento Natural de Población). Elaboración propia. 
Figura 1

Evolución de la mortalidad atribuible al tabaco por grandes causas. Islas Canarias (1975-1994)

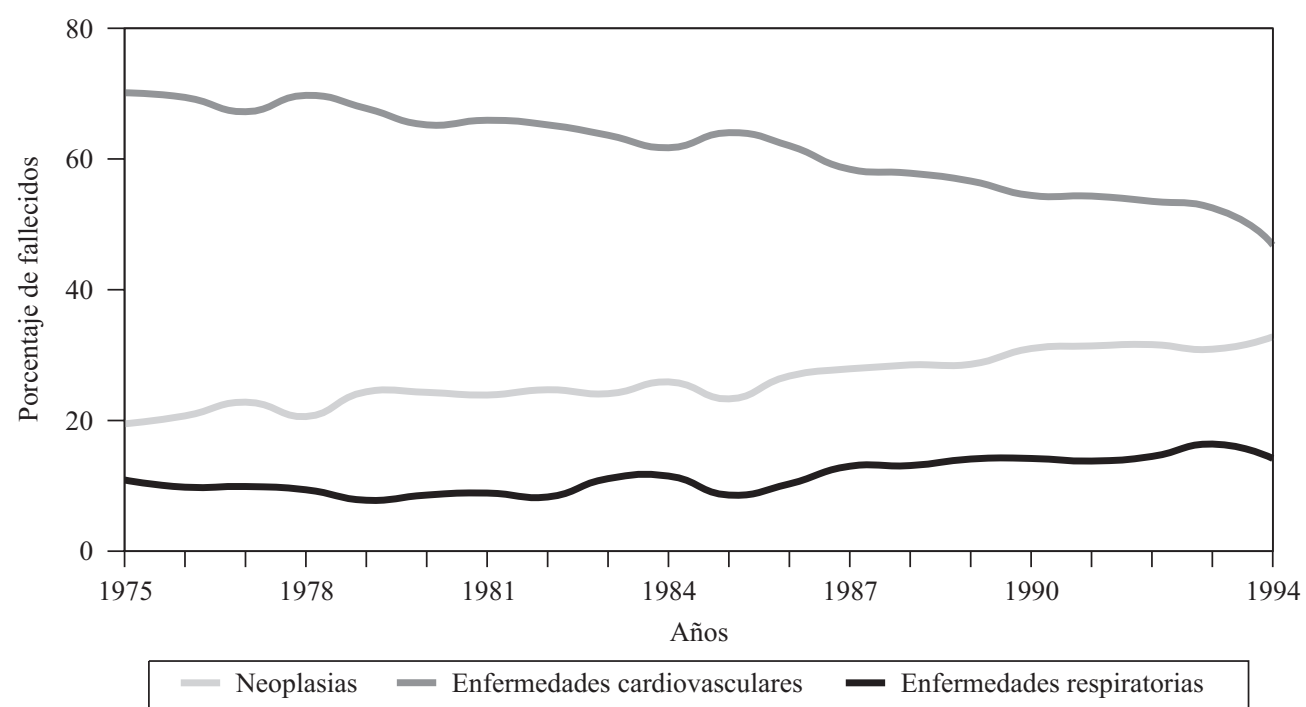

Fuente: Instituto Nacional Estadística. Movimiento Natural de Población. Elaboración propia.

Figura 2

Evolución de la mortalidad atribuible al tabaco. Islas Canarias (1975-1994)

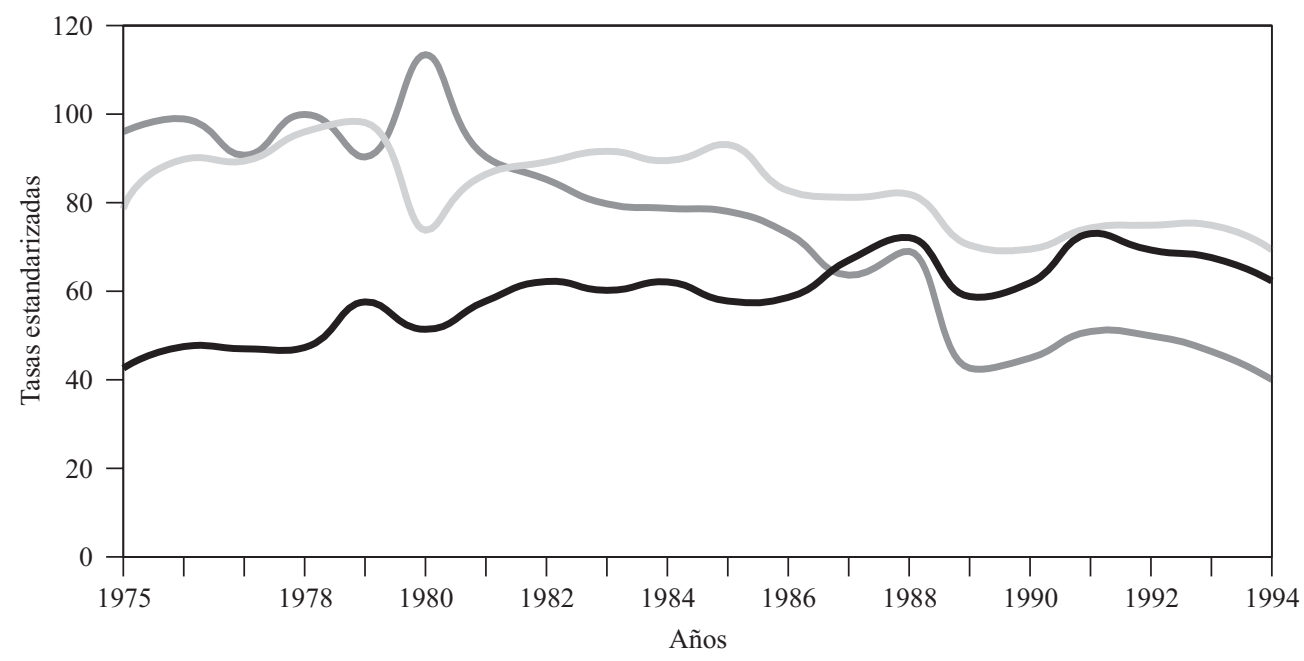

- Neoplasia de Traquea, Bronquios y Pulmón - Cardiopatía Isquemica - Enfermedad Cerebro-vascular

Fuente: Instituto Nacional Estadística. Movimiento Natural de Población. Elaboración propia.

Rev Esp Salud Pública 2001, Vol. 75, N. ${ }^{\circ} 1$ 
Tabla 3

Número y porcentaje de fallecidos atribuibles al tabaco en personas de 35 y más años, según sexo. Islas Canarias (1975 y 1994)

\begin{tabular}{|c|c|c|c|c|c|c|c|}
\hline \multirow{2}{*}{ Causas atribuibles } & \multirow{2}{*}{ CIE 8. ${ }^{a}-9^{a}$} & \multicolumn{2}{|c|}{ TOTALES } & \multicolumn{2}{|c|}{ HOMBRES } & \multicolumn{2}{|c|}{ MUJERES } \\
\hline & & 1975 & 1994 & 1975 & 1994 & 1975 & 1994 \\
\hline NEOPLASIAS & & $303(19,5)$ & $747(33,0)$ & $269(23,5)$ & $666(38,9)$ & $34(8,3)$ & $81(14,6)$ \\
\hline Labio, boca, faringe & $140-149$ & $22(1,4)$ & $92(4,0)$ & $20(1,7)$ & $87(5,0)$ & $2(0,4)$ & $5(0,9)$ \\
\hline Esófago & 150 & $42(2,7)$ & $56(2,4)$ & $33(2,8)$ & $50(2,9)$ & $9(2,2)$ & $6(1,0)$ \\
\hline Páncreas & 157 & $11(0,7)$ & $43(1,9)$ & $8(0,7)$ & $26(1,5)$ & $3(0,7)$ & $17(3,0)$ \\
\hline Laringe & 161 & $28(1,8)$ & $70(3,0)$ & $27(2,3)$ & $68(3,9)$ & $1(0,2)$ & $2(0,3)$ \\
\hline Tráquea, pulmón & 162 & $168(10,8)$ & $413(18,6)$ & $153(13,3)$ & $376(22,0)$ & $15(3,6)$ & $37(6,6)$ \\
\hline Cuello útero & 180 & - & $7(0,3)$ & - & - & 0 & $7(1,2)$ \\
\hline Vejiga & 188 & $25(1,6)$ & $53(2,3)$ & $22(1,9)$ & $47(2,7)$ & $3(0,7)$ & $6(1,0)$ \\
\hline Riñón & 189 & $7(0,4)$ & $13(0,5)$ & $6(0,5)$ & $12(0,7)$ & $1(0,2)$ & $1(0,1)$ \\
\hline ENF. CARDIOVASCULARES & & $1.089(70,2)$ & $1.193(52,7)$ & $767(67,0)$ & $801(46,9)$ & $322(79,1)$ & $392(70,7)$ \\
\hline Enfermedad hipertensiva & $\begin{array}{l}(400-404) \\
(401-405)\end{array}$ & $10(0,6)$ & $40(1,7)$ & $6(0,5)$ & $23(1,3)$ & $4(0,9)$ & $17(3,0)$ \\
\hline Cardiopatía Isquémica & $410-414$ & & & & & & \\
\hline $35-64$ & & $132(8,5)$ & $175(7,7)$ & $115(10,0)$ & $149(8,7)$ & $17(4,1)$ & $26(4,6)$ \\
\hline$>=65$ & & $191(12,3)$ & $297(13,1)$ & $132(11,5)$ & $198(11,5)$ & $59(14,4)$ & $99(17,8)$ \\
\hline Otras Enfermedades & $390-398,415$ & $208(13,4)$ & $305(13,4)$ & $141(12,3)$ & $189(11,0)$ & $67(16,4)$ & $116(20,9)$ \\
\hline Cardiovasculares & $417,420-429$ & & & & & & \\
\hline Enfermedad Cerebrovascular & $430-438$ & & & & & & \\
\hline $35-64$ & & $102(6,5)$ & $67(2,9)$ & $68(5,9)$ & $44(2,5)$ & $34(8,3)$ & $23(4,1)$ \\
\hline$>=64$ & & $292(18,8)$ & $224(9,9)$ & $203(17,7)$ & $141(8,2)$ & $89(21,()$ & $83(14,9)$ \\
\hline Ateroesclerosis & 440 & $115(7,4)$ & $31(1,3)$ & $70(6,1)$ & $13(0,7)$ & $45(11,0)$ & $18(3,2)$ \\
\hline Aneurisma Aórtico & 441 & $3(0,1)$ & $42(1,8)$ & $3(0,2)$ & $38(2,2)$ & 0 & $4(0,7)$ \\
\hline $\begin{array}{l}\text { Otras Enf. Arteriales y de los Va- } \\
\text { sos Capilares }\end{array}$ & $442-448$ & $36(2,3)$ & $12(0,5)$ & $29(2,5)$ & $6(0,3)$ & $7(1,7)$ & $6(1,0)$ \\
\hline ENF. RESPIRATORIAS & & $159(10,2)$ & $322(14,2)$ & $108(9,4)$ & $241(14,1)$ & $51(12,5)$ & $81(14,6)$ \\
\hline Neumonía/Influenza & $480-487$ & $70(4,5)$ & $86(3,8)$ & $48(4,2)$ & $51(2,9)$ & $22(5,4)$ & $35(6,3)$ \\
\hline Bronquitis/Enfisema & $491-492$ & $59(3,8)$ & $26(1,1)$ & $36(3,1)$ & $23(1,3)$ & $23(5,6)$ & $3(0,5)$ \\
\hline $\begin{array}{l}\text { Obstrucción Crónica de Vías } \\
\text { Respiratorias }\end{array}$ & 496 & - & $198(8,7)$ & - & $160(9,3)$ & - & $38(6,8)$ \\
\hline Otras Enfermedades Respiratorias & $010-012,493$ & $30(1,9)$ & $12(0,5)$ & $24(2,1)$ & $7(0,4)$ & $6(1,4)$ & $5(0,9)$ \\
\hline TOTAL & & 1.551 & 2.262 & 1.144 & 1.708 & 407 & 554 \\
\hline
\end{tabular}

Fuente: Instituto Nacional Estadística/Movimiento Natural Población. Madrid 1977-98. Elaboración propia

Por edades, se observa que el número de fallecidos aumenta con la edad; este fenómeno se presenta en ambos sexos. Así, el grupo de edad de 65 años y más, es el que presenta más fallecidos.

Durante el período 1975-1994 se observa un aumento promedio anual del $16,0 \%$ de la obstrucción de las vías aéreas (496), del 9,3\% del aneurisma aórtico (441), del $4,7 \%$ de la enfermedad hipertensiva (401-404) y del 3,8\% neoplasia de labio, boca, faringe (140-149) y una disminución promedio anual del 5,0\% de la enfermedad cerebro-vascular (430-438), del $5,9 \%$ de la bronquitis/ enfisema (491-492) y de la arteriosclerosis (440) del 6,9\%. En el sexo masculino el aumento promedio 
anual del $16 \%$ en la obstrucción de vías aéreas (496) y el 9,4\% del aneurisma aórtico (441) y el descenso del 7,4\% de la arteriosclerosis (440) y de $5,2 \%$ de la bronquitis/enfisema (491-492). Y en el sexo femenino, como más destacado, el aumento promedio anual del $15,5 \%$ de la obstrucción de las vías aéreas (496) y el 8,2\% del aneurisma aórtico (441) y el 5,6\% en la enfermedad hipertensiva (401-404) (tabla 4).

\section{DISCUSIÓN}

El objetivo del estudio es cuantificar los efectos del tabaco sobre la mortalidad de las Islas Canarias en el período 1975-1994. Nos encontramos con algunos problemas como consecuencia de las numerosas in- vestigaciones en España e Islas Canarias sobre prevalencias del consumo de tabaco en las islas, al incluir diferentes resultados según las encuestas fueran de ámbito estatal o regional. Así, en la Encuesta Nacional de Salud (1989) y en la Encuesta Nacional de la Salud (1993) se observó una prevalencia del consumo de tabaco para las Islas Canarias de $38,2 \%$ y $37 \%$ respectivamente; en cambio en la Encuesta de Salud de Canarias (1990) y la Encuesta de Salud de Canarias (1997) los resultados fueron de $30,3 \%$ y $33 \%$ respectivamente. Además, los cálculos que se hicieron para conocer la proporción de varones y mujeres fumadoras en la década de los 70 fueron sólo estimaciones para todo el Estado y no para las Islas Canarias ${ }^{13}$. Todo esto influiría en el número de muertes atribuibles al tabaco en cada año.

Tabla 4

Cambio porcentual medio anual de las tasas ajustadas de mortalidad atribuible al consumo de Tabaco en personas de 35 años y más, según sexo. Islas Canarias (1975-1994)

\begin{tabular}{|c|c|c|c|}
\hline Causa de Muerte & $\begin{array}{c}\text { Total } \\
\text { CMA (IC 95\%) }\end{array}$ & $\begin{array}{c}\text { Masculino } \\
\text { CMA (IC 95\%) }\end{array}$ & $\begin{array}{c}\text { Femenino } \\
\text { CMA (IC 95\%) }\end{array}$ \\
\hline \multicolumn{4}{|l|}{ NEOPLASIAS } \\
\hline Labio, Boca, Faringe (140-149) & $3,8(3,0-4,6)$ & $3,8(2,9-4,6)$ & $4,4(1,07-7,7)$ \\
\hline Esófago (150) & $-0,4(-1,2-0,3)$ & $-0,9(-0,95-0,7)$ & $-3,3(-5,6$ a $-0,9)$ \\
\hline Páncreas (157) & $2,8(1,7-3,9)$ & $2,0(0,7-3,3)$ & $5,0(2,9-7,1)$ \\
\hline Laringe (161) & $0,9(0,1-1,8)$ & $1,0(0,2-1,8)$ & $-0,6(-5,5-4,2)$ \\
\hline Tráquea, Bronquios, Pulmón (162) & $1,9(1,6-2,2)$ & $2,0(1,7-2,4)$ & $0,6(-0,4-1,7)$ \\
\hline Cuello de útero & - & - & $3,1(0,3-5,8)$ \\
\hline Vejiga (188) & $0,5(-0,2-1,4)$ & $0,3(-0,5-1,3)$ & $2,5(-0,4-5,5)$ \\
\hline Riñón (189) & $1,9(-0,03-3,8)$ & $1,8(-0,1-3,9)$ & $2,0(-4,7-8,9)$ \\
\hline \multicolumn{4}{|l|}{ ENFERMEDAD CARDIOVASCULAR } \\
\hline Enfermedad hipertensiva (401-404) & $4,7(3,4-5,9)$ & $4,2(2,7-5,8)$ & $5,6(3,5-7,8)$ \\
\hline Cardiopatía isquémica (410-414) & $-1,1(-1,4 \mathrm{a}-0,8)$ & $-1,2(-1,6 \mathrm{a}-0,9)$ & $-0,5(-1,1$ a 0,03$)$ \\
\hline Enfermedad Cerebrovascular (430-438) & $-5,0(-5,4 \mathrm{a}-4,8)$ & $-4,7(-5,0 \mathrm{a}-4,3)$ & $-5,6(-6,2$ a $-5,0)$ \\
\hline Arteriosclerosis (440) & $-6,9(-7,5 \mathrm{a}-6,3)$ & $-7,4(-8,1$ a $-6,5)$ & $-6,4(-7,4$ a $-5,3)$ \\
\hline Aneurisma Aórtico (441) & $9,3(7,6-11)$ & $9,4(7,6-11,3)$ & $8,2(2,5-14,3)$ \\
\hline \multicolumn{4}{|l|}{ ENFERMEDADES RESPIRATORIAS } \\
\hline Neumonía (480-487) & $-0,2(-0,8-0,3)$ & $0,3(-0,4-1,0)$ & $-1,2(-2,2 \mathrm{a}-0,1)$ \\
\hline Bronquitis/Enfisema (491-492) & $-5,9(-7,0 \mathrm{a}-5,3)$ & $-5,2(-6,2$ a $-4,1)$ & $-8,5(-10,3$ a $-6,6)$ \\
\hline Obstrucción Vías Aéreas (496) & $16,0(15,2-16,8)$ & $16,2(15,3-17,1)$ & $15,5(13,6-17,4)$ \\
\hline
\end{tabular}

Fuente: INE/MNP. Elaboración propia.

$\left({ }^{*}\right)$ CMA: El Cambio Medio Anual, calculado mediante modelo log-lineal.

$(* *) \quad$ IC: Intervalo de confianza del $95 \%$. 
El tabaco en las Islas Canarias en el período 1975-1994 produjo un total de 41.495 muertes, de las cuales 30.550 fueron en varones y 10.945 en mujeres; representando entre el 19\%-21\% de todos los fallecidos cada año. En España, en el período de tiempo de 1978-1992 se produjo un total de 600.000 fallecimientos, lo que representó aproximadamente el $14 \%$ de las muertes ocurridas cada año atribuidas al consumo de tabaco ${ }^{2}$. En Francia, representa el $12 \%$ del total de fallecidos ${ }^{15}$; en Cataluña (1990) el 16\% ${ }^{16}$; y en Madrid (1995) el 18\% ${ }^{3}$. El porcentaje anual de fallecidos en las Islas Canarias por el tabaco es mayor que en España, Cataluña y Madrid. En 1994 supera la barrera del $20 \%$ de los fallecidos anualmente por tabaco, cifra ésta que se considera en la actualidad como límite en los países desarrollados $^{5,6}$. En los hombres, representó el 29\% de fallecidos por tabaco, porcentaje ligeramente superior al del año 1975. Esta cifra es alta si la comparamos con Japón $(17 \%)^{7}$ y baja si la comparamos con Polonia $(50 \%)^{7}$; aunque en este caso, como en otros de países del este de Europa, parecen contribuir factores de índole laboral y contaminación aérea ${ }^{7}$. Las mujeres, han mantenido cifras que oscilan entre el $10,7 \%-12,2 \%$ en el año 1994 . Peto y col ${ }^{7}$ observaron que el riesgo para las mismas en España continúa siendo bajo. Aunque en el archipiélago se observó casi un 60\% menos de fallecimientos por consumo de tabaco en las mujeres con respecto a los hombres, en el año 1994 va incrementándose, tanto la prevalencia de fumadoras adolescentes como el número de fallecidas. En 1997 ya consumían tabaco casi el 40\% de las mujeres entre 15-44 años.

Si es altísimo el tributo que hay que pagar en España, en términos de muertes evitables $\mathrm{y}$ de sufrimiento, mayor es el tributo que hay que pagar en las Islas Canarias, ya que el porcentaje es mayor. Se pone en evidencia que las medidas legales y educativas implementadas son insuficientes para controlar el problema.

En las Islas Canarias, durante el año 1994 el número de personas fallecidas por tabaco fue mayor que las fallecidas por otras causas (el suicidio, homicidio o accidentes de tráfico), aunque éstas se producen en personas de más edad con menor influencia en los AVPP, al contrario que las causas antes mencionadas. Este fenómeno se observó también en Puerto Rico ${ }^{17}$.

La discusión sobre aspectos metodológicos en este tipo de estudios ha sido ya expuesto en trabajos anteriores ${ }^{12,13}$, en cambio, hemos encontrado algunos problemas de índole práctica con los fallecidos antes de 1980 del Instituto Nacional Estadística (INE) para algunas causas, probablemente han sido problemas de clasificación del paso de la CIE 8. a CIE 9. ${ }^{\mathrm{a}}$

En los hombres las prevalencias de consumo de tabaco han oscilado entre el $74 \%$ y el $52 \%$ con tendencia a la estabilización; en la mujer, entre el 15\% y $30 \%$ con tendencia a aumentar. La exposición de la población al tabaco, sobre todo en la mujer aún no ha alcanzado su máxima expresión, en cuanto a sus consecuencias (mortalidad y morbilidad). Es de prever un aumento en las enfermedades que se derivan del tabaco de continuar la tendencia. Hasta el año 1994 el porcentaje de fallecidos por enfermedades cardiovasculares $(52,5 \%)$ era mayor que las neoplasias $(32,9 \%)$; lo normal es que al finalizar la década de los 90 esta tendencia se invierta por el incremento anual que están teniendo las neoplasias y el descenso de las enfermedades cardiovasculares, menos acelerado en las mujeres, como ha sucedido en España a partir del año $1986^{3}$ (figura 1). Ahora bien, si lo analizamos por causas específicas, observamos que la neoplasia de tráquea, bronquios y pulmón supera a la enfermedad cerebro-vascular desde el año 1987 (figura 2). Dicho fenómeno es también reflejado por González Enríquez ${ }^{3}$ en el contexto del España desde el año 1981.

La neoplasia de tráquea, bronquios y pulmón (162) es la causa específica que mayor porcentaje de defunciones produjo en varones $(21,9 \%)$ en el año 1994 . Una de cada cuatro muertes atribuibles al tabaco se pro- 
ducen por esta causa en las Islas Canarias, a diferencia del resto del estado español donde es una de cada tres ${ }^{3}$. En la mujer todavía el crecimiento es mínimo (0,6\% anual) y la proporción de defunciones es del 6,6\% con relación al total en el año 1994, aunque según Banegas ${ }^{18}$ predicen una futura epidemia de neoplasia de pulmón en la mujer en las próximas décadas, si no se toman medidas preventivas, debido a la importante incorporación de un mayor tanto por ciento de jóvenes fumadoras.

Aunque los datos sobre las defunciones atribuibles al consumo de tabaco en las Islas Canarias constituyen un punto de partida y de apoyo para establecer actividades de lucha contra el aumento del tabaquismo, hay que producir otro tipo de investigaciones científicas para reforzar estas evidencias y demostrar el efecto concreto del tabaco en la salud de la población canaria (morbilidad, bajo peso al nacer, etcétera).

\section{BIBLIOGRAFÍA}

1. CDC. State-specific prevalence among adults of current cigarette smoking and smokeless tobacco use and per capita tax-paid sales of cigarettes-United States, 1997. MMWR 1998; 47 (43): 922-926.

2. González Enríquez J, Villar Álvarez F, Banegas Banegas JR, Rodríguez Artalejo F, Martín Moreno JM. Tendencia de la mortalidad atribuible al tabaquismo en España 1978-1992: 600.000 muertes en 15 años. Med Clin (Barc) 1997; 109: 577-582.

3. Comunidad de Madrid. Vigilancia del consumo de tabaco y sus efectos para la salud en la Comunidad de Madrid. Bol Epidemiol Comunidad de Madrid 1997; 5 (11): 3-27.

4. Santana Armas JF, Orengo JC, Santana Armas Y, Lorenzo P, Serra Majem L. Mortalidad atribuible al tabaquismo en Canarias. Canarias Médica 1998; 13: 15-19.

5. Nelson DE et al. Surveillance for smoking-attributable mortality and years of potencial life lost, by State-United States,1990. MMWR 1994; 43 (SS-1): 1-8.

Rev Esp Salud Pública 2001, Vol. 75, N. ${ }^{\circ} 1$
6. CDC. Smoking-attributable mortality-Mexico, 1992. MMWR 1995; 44 (19): 379-381.

7. Peto R, López AD, Boreham J, Thun M, Heath C Mortality from smoking in developed countries 1950-2000. Oxford: Oxford University Press; 1994.

8. Ministerio de Sanidad y Consumo. Encuesta Nacional de Salud de España 1993. Madrid: Ministerio de Sanidad y Consumo; 1995.

9. Ministerio de Sanidad y Consumo. Encuesta Nacional de Salud de España 1987. Madrid: Ministerio de Sanidad y Consumo; 1989.

10. Consejería de Sanidad, Trabajo y Servicios Sociales del Gobierno de Canarias. Encuesta de Salud de Canarias; 1990. (Documento mimeografiado).

11. Servicio Canario de Salud. Encuesta de Salud de Canarias 1997. Santa Cruz de Tenerife: Servicio Canario de Salud; 1998.

12. US Department of Health and Human Services. Reducing the Health Consequences of Smoking. 25 years of progress. A report of the Surgeon $\mathrm{Ge}-$ neral, 1989. Rockville, Maryland: US DHHS, PHS, CDC, Center for Chronic Disease Prevention and Health Promotion, Office on Smoking and Health, 1989. DHHS Publication N. ${ }^{\circ}$ (CDC) 89-8411; 1989.

13. González Enríquez J, Rodríguez Artalejo F, Martín Moreno J, Banegas Banegas JR, Villar Álvarez F. Muertes atribuibles al consumo de tabaco en España. Med Clin (Barc) 1989; 92: 15-18.

14. McCullagh P, Nelder JA. Generalized linear models. 2. ${ }^{a}$ ed. Londres: Chapman and Hall; 1989.

15. Hill C. Trends in tobacco and consequences on health in France. Prev Med 1998; 27 (4): 514-519.

16. Serra Majem Ll, de Cambra S, Saltó E, Roura E, Rodríguez F, Vallbona C, Salleras L, Consejo antitabaco. Med Clin (Barc) 1994; 102 Supl 1: 109-117.

17. Dietz VJ, Novotny TE, Rigau-Perez JG, Shultz JM. Mortalidad atribuible al tabaquismo, años de vida potencial perdidos y costos directos para la atención de salud en Puerto Rico, 1983. Bol Sanit Panam 1991; 110 (5): 378-389.

18. Banegas Banegas JR, Rodríguez Artalejo F, Martín Moreno JM, González Enríquez J, Villar Álvarez F, Guasch Aguilar A. Proyección del impacto tabáquico sobre la salud de la población española y de los beneficios potenciales de su control. Med Clin (Barc) 1993; 101: 644-649. 